\title{
Performance evaluation of Frame Slotted-ALOHA with Intra-Frame and Inter-Frame Successive Interference Cancellation
}

\author{
A. Hernández ${ }^{\dagger}$, F. Vázquez-Gallego*, L. Alonso ${ }^{\dagger}$, and J. Alonso-Zarate* \\ ${ }^{*}$ Centre Tecnològic de Telecomunicacions de Catalunya (CTTC), Castelldefels, Barcelona, Spain \\ \{rancisco.vazquez, jesus.alonso\}@cttc.es \\ $\dagger$ Universitat Politècnica de Catalunya (UPC), Castelldefels, Barcelona, Spain \\ ana.cristina.hernandez@estudiant.upc.edu, luisg@tsc.upc.edu
}

\begin{abstract}
Machine-to-Machine (M2M) networks allow enddevices to communicate without human intervention. Due to the high density of M2M networks, efficient Medium Access Control (MAC) protocols are required to manage the access to the channel. In this paper, we consider an M2M area network composed of hundreds of end-devices that periodically transmit data to a gateway. We evaluate the performance of two Medium Access Control (MAC) protocols based on Frame Slotted-ALOHA (FSA) with Successive Interference Cancellation (SIC): Intra-frame SIC-FSA and Inter-frame SIC-FSA. By means of computer-based simulations, we have compared the delay and energy performance with respect to conventional FSA. Results show that the average delay can be reduced in a $78 \%$ and 94\% by using Intra-frame SIC-FSA and Inter-frame SIC-FSA, respectively, and the average energy consumed per end-device is reduced in $18 \%$ by using Intra-frame SIC-FSA, while Interframe SIC-FSA decreases the energy consumed per end-device in a $22 \%$.
\end{abstract}

Index Terms-Successive Interference Cancellation, Frame Slotted-ALOHA, M2M networks, data collection.

\section{INTRODUCTION}

Machine-to-Machine (M2M) networks allow communications between a huge number of end-devices without human intervention. In order to reduce the maintenance costs of $\mathrm{M} 2 \mathrm{M}$ networks, it is crucial to prolong the lifetime of the end-devices. Consequently, energy efficiency is one of the main challenges in the deployment of M2M networks, and the communication protocols need to be tailored to minimize energy consumption. In this paper, we focus on data collection scenarios where a group of end-devices transmit data to a gateway periodically upon request. While the traffic load generated by each end-device may be low, the total number of end-devices that may attempt to get access simultaneously can be large. Therefore, efficient Medium Access Control (MAC) protocols are needed in order to manage the access to the wireless channel and reduce collisions.

This work was supported by the Research Projects ADVANTAGE (FP7-MCA-607774), CellFive (TEC2014-60130-P), P2P-SmarTest (H2020646469), GEOCOM (TEC2011-27723-C02-01) and by the Generalitat de Catalunya under Grant 2014 SGR 1551.
Random access protocols are typically used in M2M networks because their distributed operation makes them easy to be implemented in low-cost and simple end-devices. In fact, many standards used in M2M (e.g., IEEE 802.11, IEEE 802.15.4, or ISO-18000-7) rely on random access protocols such as Carrier Sense Multiple Access (CSMA), ALOHA, and Frame Slotted-ALOHA (FSA). Unfortunately, random access suffers from degraded performance when the number of enddevices increases or when the traffic load is very high. Since the end-devices must compete for the channel, the higher the number of end-devices, the higher the probability of collision, thus increasing the delay and energy consumption.

Recently, several research works, e.g., [1]-[7], have shown that collisions can be resolved by using Successive Interference Cancellation (SIC) techniques that improve the performance of ALOHA-based protocols. In order to perform SIC, each end-device transmits a number of replicas of a data packet in different slots. A gateway (or coordinator) might potentially solve collisions if the replicas of the collided packets have been decoded in different slots.

The works in [1] and [2] proposed ALOHA-based protocols with SIC named Contention Resolution Diversity SlottedALOHA (CRDSA) and Irregular Repetition Slotted-ALOHA (IRSA), respectively. Results show that CRDSA and IRSA increase the system throughput in a $20 \%$ with respect to slottedALOHA. Similar results were obtained in [3] considering variants of FSA with SIC in Radio Frequency Identification (RFID) systems.

The performance enhancement provided by the SIC-FSA protocol has been evaluated in terms of delay and energy consumption in M2M networks for data collection [5]. In SICFSA, also referred to as Intra-frame SIC FSA, the coordinator executes the SIC algorithm within each frame. Results show that Intra-frame SIC-FSA can reduce the delay and energy consumption of the gateway in more than $50 \%$, and the energy consumed per end-device in more than $10 \%$, with respect to FSA. While the research in [5] considers that each enddevice transmits a fixed number of replicas of every data packet in each frame, the work in [2] analyzes the throughput of Intra-frame SIC-FSA using a random number of replicas. 
Results show that the throughput is maximized by using certain probability density functions.

The works in [6] and [7] proposed the Inter-frame SIC technique on Pseudo-Random ALOHA. In Inter-frame SIC, the replicas of a data packet which are decoded in a given frame are used to resolve collisions that were caused by the other replicas of the same data packet in slots of previous frames. The works in [6] and [7] analyzed and compared the benefits of the Inter-frame SIC technique, in terms of throughput, with respect to Intra-frame SIC. Results show that Inter-frame SIC slightly improves the throughput with respect to Intra-frame SIC.

Up to our knowledge, the evaluation of Inter-frame SIC and Intra-frame SIC in FSA using random numbers of replicas has never received attention in data collection scenarios. This is the main motivation of this paper, where we present the performance evaluation of Intra-frame SIC-FSA and Interframe SIC-FSA, and compare with conventional FSA, in terms of delay and energy consumed by the coordinator and enddevices. The remainder of this paper is organized as follows. In Section II, we describe the system model. In Section III, we describe the MAC protocols. Section IV is devoted to the description of the energy and delay models. In Section V, we evaluate the performance of the protocols through computerbased simulations. Finally, Section VI concludes the paper.

\section{System ModeL}

We consider a wireless network composed of $n$ end-devices which periodically transmit data to a coordinator (also referred to as gateway) in Data Collection Rounds (DCR). Every DCR starts when the coordinator broadcasts a Request for Data (RFD) packet. We assume that each end-device has exactly one data packet to transmit in every DCR. In addition, we assume that all the end-devices are listening to the channel when the coordinator transmits a RFD. After decoding a RFD, the enddevices get synchronized to a common time frame pattern and contend for the channel according to the rules of the adopted MAC protocol, as described in Section III.

The RFD packet is followed by a sequence of frames divided into $m$ slots. The duration of a slot is fixed and equal to the duration of a data packet. In every frame, the enddevices choose randomly the slots to transmit its data packet without performing carrier sense. Consequently, each slot can be in one of three states: $(i)$ empty, when no end-device has transmitted in this slot; $(i i)$ success, when only one end-device has selected this slot to transmit its data packet; and (iii) failure, when one or more end-devices have transmitted in this slot but none of the data packets can be decoded by the coordinator due to collision or channel error. We assume that all data packets are transmitted without errors induced by the channel and there is no capture effect. Thus, no data packet involved in a collision can be decoded by the coordinator. The inclusion of capture effect and transmission errors constitutes part of our future work. In addition, we assume that the coordinator has perfect knowledge of the wireless channel with each end-device.
At the end of each frame the coordinator sends a Feedback Packet (FBP) to inform the end-devices about the data packets that have been successfully decoded. Once an end-device has succeeded in transmitting its data packet in a given frame, it switches into sleep mode in order to save energy. However, the end-devices that have not succeeded will contend again in subsequent frames. A DCR finishes when the coordinator has decoded the data packets of all the end-devices.

The coordinator and the end-devices can be in fives modes of operation: $(i)$ transmitting a packet, $(i i)$ receiving a packet, (iii) idle listening, $(i v)$ standby, and $(v)$ sleeping. The associated power consumptions are $\rho_{t x}, \rho_{r x}, \rho_{\sigma}, \rho_{\text {stby }}$, and $\rho_{\text {sleep }}$, respectively. We assume that $\rho_{r x}=\rho_{\sigma}$. The Inter-Frame Spaces (IFS) are included in every frame to compensate propagation delays and the time required by the end-devices to switch between reception and transmission modes.

\section{Medium AcCess Control Protocols}

In this section, we describe the MAC protocols considered: FSA, Intra-frame SIC-FSA, and Inter-frame SIC-FSA. In FSA, each device transmits its data packet in one randomly selected slot of every frame until it succeeds. In Intra-frame SICFSA and Inter-frame SIC-FSA the $i$-th end-device transmits $k_{i}$ replicas of its data packet in $k_{i}$ randomly selected slots of every frame, where $k_{i} \in\{1,2, \ldots, k\}$ is a random number that follows a given probability density function (PDF), and $k$ is the maximum number of replicas. The MAC header of each replica of a data packet includes a pointer to the position of the other $k_{i}-1$ replicas.

\section{A. Frame Slotted-ALOHA with Intra-frame SIC}

In Intra-frame SIC-FSA, the coordinator uses the information from every successfully decoded replica to cancel the interference that the remaining replicas of the same data packet may cause in other slots of the current frame. The coordinator subtracts the decoded packet to the signal received in the slots where the replicas have caused collisions. Due to the fact that every decoded data packet can be used to decode other collided packets, the SIC process is iterated until the coordinator is unable to decode more data packets, i.e, there are only empty slots or slots with collisions that cannot be solved. Figure 1 shows an example of a DCR using Intra-frame SIC-FSA with $m=6$ slots and $n=5$ end-devices that can transmit a random number of replicas with $k \in\{1,2,3\}$. In this example, the DCR finishes after four SIC iterations within one frame. In the first iteration of the SIC process, the coordinator decodes the data packet of end-device 2 in slot 2 (packet 2) and the data packet transmitted by end-device 4 in slot 5 (packet 4). In the second iteration, the coordinator subtracts packet 2 to the signal received in slot 3 and packet 3 is decoded. In the third iteration, the coordinator decodes one replica of packet 5 by subtracting packets 2,3 and 4 to the signal received in slot 4 . Finally, the fourth iteration allows the coordinator to decode packet 1 by subtracting packet 5 to the signal received in slot 1 . 


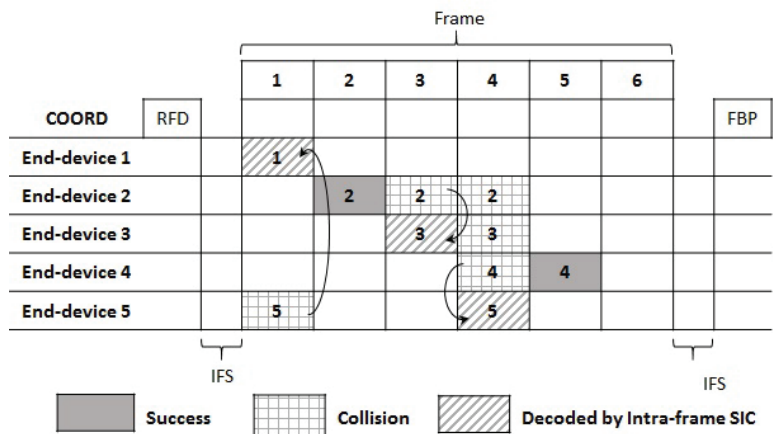

Figure 1. Example of operation of Intra-frame SIC-FSA.

\section{B. Frame Slotted-ALOHA with Inter-Frame SIC}

In Inter-frame SIC-FSA, the SIC process is executed by using the information of a successfully decoded replica in a given frame to cancel the interference that other replicas of the same data packet may cause in slots of previous frames. The Inter-frame SIC process is divided into three sub-processes.

Firstly, the coordinator executes Intra-frame SIC at the end of frame $j$. If there are slots with unsolved collisions, the coordinator subtracts the decoded packets to the collision signals from previous frames by following the Backward and Forward SIC algorithms. The parameter $s$ denotes the number of frames in which Backward and Forward SIC are executed.

Secondly, the coordinator executes the Backward SIC algorithm. In this stage, the packets decoded in frame $j$ are used to resolve collisions in frame $j-1$. Subsequently, the packets decoded in frame $j-1$ by means of Backward SIC are used to resolve collisions in frame $j-2$. This process is performed iteratively and stops in frame $j-s+1$. Therefore, the coordinator has to store in memory all the signals received in each collided slot of every $s$ consecutive frames and knows the position of the replicas of each data packet in every frame.

Thirdly, considering that the Backward SIC algorithm ends in frame $j-2$, i.e., $s=3$, the Forward SIC algorithm uses the information of successfully decoded packets in $j-2$ to resolve collisions in frames $j-1$ and $j$.

Figure 2 shows an example of a DCR using Inter-frame SIC-FSA with $s=2$ frames, $m=6$ slots, and $n=6$ enddevices that transmit a fixed number of replicas $k=2$. In frame $j-1$, the coordinator decodes packet 4 in slot 4 , and stores the signals of the collided slots in frame $j-1$. The Intra-frame SIC algorithm is executed in frame $j-1$, but only packet 4 has been decoded. In frame $j$, the coordinator decodes packet 1 in slot 4 . Using packet 1 decoded in frame $j$, the coordinator performs Backward SIC and consequently solves the collisions in slots 2 and 3 of frame $j-1$, thus decoding packets 2 and 3. Then, Forward SIC is executed, using packets 2 and 3 decoded in frame $j-1$ to solve collisions in frame $j$ and decode packets 5 and 6 , respectively.

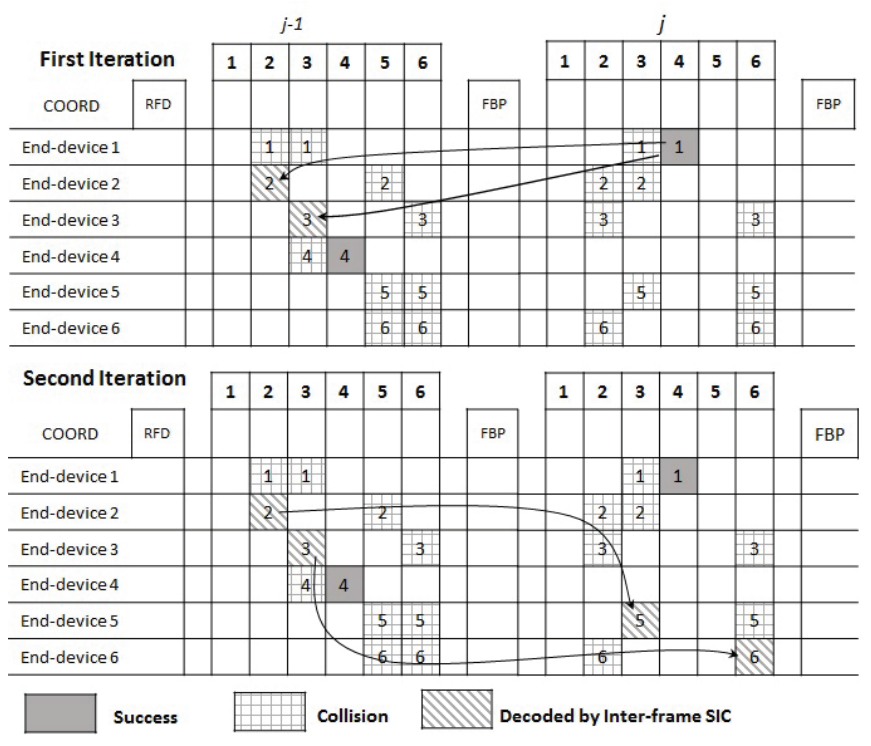

Figure 2. Example of operation of Inter-frame SIC-FSA.

\section{Delay And Energy Consumption Models}

In this section, we formulate the delay and energy consumption models of FSA, Intra-frame SIC-FSA and Inter-frame SIC-FSA. These models will be used to compute the average delay to finish a DCR, the average energy consumed by the coordinator and the average energy consumed per end-device.

The average delay required to finish a DCR can be formulated as:

$$
\overline{T_{D C R}}=\overline{C F}\left(m T_{S L O T}+2 T_{I F S}+T_{F B P}\right),
$$

where $T_{S L O T}, T_{I F S}$ and $T_{F B P}$ are the duration of a slot, an IFS and the time required to transmit a FBP, respectively, and $\overline{C F}$ is the average number of frames required to complete a DCR.

The average energy consumed by the coordinator to successfully receive one data packet from each end-device during a DCR is denoted by $\overline{E_{\text {coord }}}$. The coordinator executes the following operations in every frame: $(i)$ it listens to the channel in the $m$ slots and stores in memory the signals received in each slot, (ii) it performs a number of SIC iterations, and (iii) it transmits a FBP. Since we focus on the energy devoted to communications (i.e., we do not consider the energy required to execute the SIC process), $\overline{E_{\text {coord }}}$ can be formulated as:

$$
\overline{E_{\text {coord }}}=\overline{C F}\left(m \rho_{r x} T_{S L O T}+2 \rho_{\sigma} T_{I F S}+\rho_{t x} T_{F B P}\right),
$$

The average energy consumed per end-device is denoted by $\overline{E_{\text {device }}}$. An end-device executes the following operations in every frame as long as it has not succeeded: $(i)$ it transmits $k_{i}$ replicas of the data packet in $k_{i}$ of the $m$ slots, $(i i)$ it keeps in standby mode for the remaining $m-k_{i}$ slots, and (iii) it receives a $\mathrm{FBP}$ from the coordinator. 
The energy consumed by an end-device in a frame where it contends can be formulated as

$$
\begin{gathered}
E_{t x}=\overline{k_{i}} \rho_{t x} T_{S L O T}+\left(m-\overline{k_{i}}\right) \rho_{s t b y} T_{S L O T}+ \\
2 \rho_{\sigma} T_{I F S}+\rho_{r x} T_{F B P},
\end{gathered}
$$

where $\overline{k_{i}}$ represents the average number of replicas that an end-device transmits in every frame.

Once an end-device succeeds in transmitting its data packet in a given frame, it sleeps for the remaining of the DCR. The energy consumption of an end-device during a frame where it sleeps can be formulated as

$$
E_{\text {sleep }}=\rho_{\text {sleep }}\left(m T_{S L O T}+2 T_{I F S}+T_{F B P}\right) .
$$

Finally, the average energy consumed per end-device can be formulated as:

$$
\overline{E_{\text {device }}}=\overline{C F_{t x}} E_{t x}+\overline{C F_{\text {sleep }}} E_{\text {sleep }},
$$

where $\overline{C F_{t x}}$ is the average number of frames where an enddevice contends and $\overline{C F_{\text {sleep }}}$ is the average number of frames where the end-device sleeps until the DCR ends.

All the parameters in the expressions of $\overline{T_{D C R}}, \overline{E_{\text {coord }}}$ and $\overline{E_{\text {device }}}$ have deterministic values, except for the values of $\overline{C F}, \overline{C F_{t x}}$ and $\overline{C F_{\text {sleep }}}$ which are obtained by computer-based simulations in the next section.

\section{Performance Evaluation}

The performance of FSA, Intra-frame SIC-FSA, and Interframe SIC-FSA is evaluated and compared in this section by means of computer-based simulations using MATLAB. The results of 1,000 simulation samples have been averaged to obtain the delay and energy consumption results for each protocol. The system parameters are summarized in Table 1. They were selected according to the IEEE 802.11 standard [8] and from the technical specifications of the RN-131 module [9], a low-power Wi-Fi device from Roving Networks. The FBP packet has been set to attach 16 bits per slot that include the information about the status of each slot and the identification of the end-devices whose data packet has been decoded. The payload length of each data packet has been set to 1024 bytes.

For Intra-frame SIC-FSA and Inter-frame SIC-FSA, we use different PDFs for the random number of replicas in every frame. We consider the irregular PDFs proposed in [2] to improve the performance of the ALOHA-based protocols. The PDFs are formulated in Equation (6), where each coefficient of the polynomials represents the probability that an end-device

Table I

SYSTEM PARAMETER

\begin{tabular}{|c|c|c|c|}
\hline Parameter & Value & Parameter & Value \\
\hline \hline MAC header & 30 bytes & Data-rate & $54 \mathrm{Mbps}$ \\
\hline Data payload & 1024 bytes & $\mathrm{T}_{\text {SLOT }}$ & $176.74 \mu \mathrm{s}$ \\
\hline FBP payload & 16 bits/slot & $\mathrm{T}_{\mathrm{FBP}}$ & $\mathrm{FBP} \cdot 8 /$ data-rate \\
\hline CRC & 4 bytes & $\mathrm{T}_{\mathrm{IFS}}$ & $10 \mu \mathrm{s}$ \\
\hline$\rho_{t x}$ & $210 \mathrm{~mA} \cdot 3 \mathrm{~V}$ & $\rho_{\text {sleep }}$ & $3.4 \mu \mathrm{W}$ \\
\hline$\rho_{r x}=\rho_{\sigma}$ & $40 \mathrm{~mA} \cdot 3 \mathrm{~V}$ & $\rho_{\text {stby }}$ & $15 \mathrm{~mA} \cdot 3 \mathrm{~V}$ \\
\hline
\end{tabular}

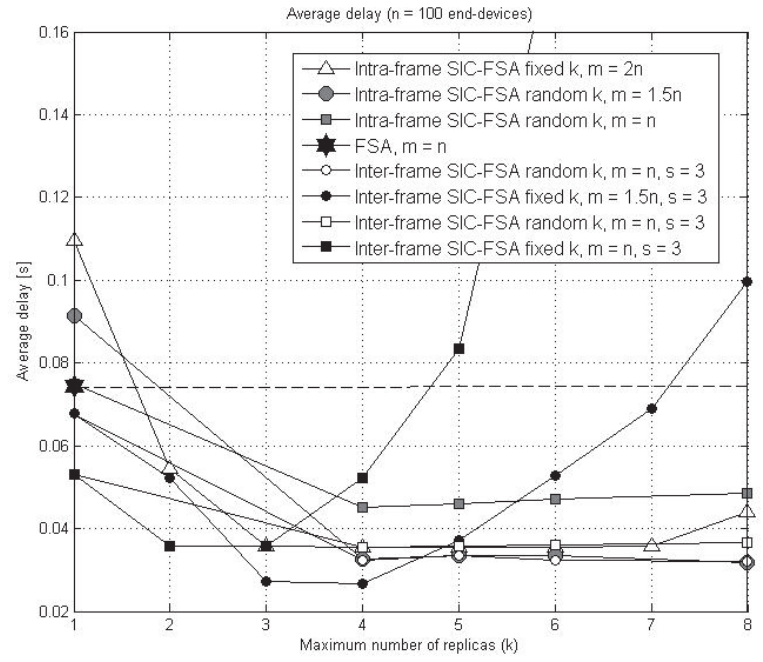

Figure 3. Average delay using FSA, Intra-frame SIC-FSA, and Inter-frame SIC-FSA over the maximum number of replicas.

selects a number $i$ of replicas, where $i$ is indicated by the exponent of each term and the maximum number $k$ of replicas is defined by the order of the polynomials.

$$
\mathrm{PDF}=\left\{\begin{array}{ccc}
0.5102 x^{2}+0.48982 x^{4} & , \text { if } & k=4 \\
0.5631 x^{2}+0.0436 x^{3}+0.3933 x^{5} & , \text { if } & k=5 \\
0.5465 x^{2}+0.1623 x^{3}+0.2912 x^{6} & , \text { if } & k=6 \\
0.5 x^{2}+0.28 x^{3}+0.22 x^{8} & \text {, if } & k=8
\end{array}\right.
$$

In the following sections, we evaluate the average delay and energy consumed by the coordinator and the end-devices as a function of the maximum number $k$ of replicas and frame length $m$, considering the selection of a random and fixed number of replicas. Finally, we evaluate the delay and energy consumption as a function of the number $n$ of end-devices.

\section{A. Optimum Number of Slots per Frame and Number of} Replicas

Figure 3, Figure 4 and Figure 5 show the average delay, the average energy consumed by the coordinator and the average energy consumed per end-device, respectively. They have been evaluated as a function of the maximum number $k$ of replicas, considering $n=100$ end-devices, 3 different frame lengths $(m=n, m=1.5 n$ and $m=2 n)$, and $s=3$ for Inter-frame SIC-FSA.

It is worth to evaluate the performance of Intra-frame SIC-FSA and Inter-frame SIC-FSA when only one replica is transmitted, i.e., $k=1$. Due to the fact that Intra-frame SICFSA requires more than one replica to resolve collisions in a frame, it does not improve the performance with respect to FSA if $k=1$. On the contrary, Inter-frame SIC-FSA outperforms FSA because it can resolve collisions between consecutive frames, although only one replica is sent.

As it can be observed in Figure 3, if the number of replicas is fixed, i.e., it is not random, the delay increases when the 


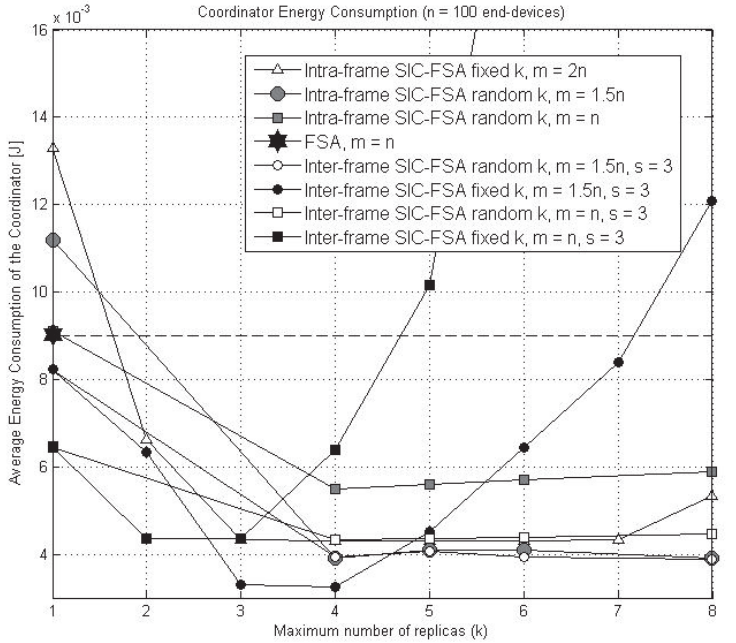

Figure 4. Average energy consumed by the coordinator using Intra-frame and Inter-frame SIC-FSA over the maximum number of replicas.

number of replicas increases for both Intra-frame SIC-FSA and Inter-frame SIC-FSA. On the contrary, the use of a random number of replicas keeps the delay almost insensitive to the maximum number of replicas. This fact can be explained by computing the average the number of replicas transmitted in every frame. Results show that the average number of replicas remains in the range [3,4], therefore, there is not a significant variation in delay.

The work in [5] demonstrated that the transmission of a fixed number of replicas can minimize delay when the frame length is set to $m=2 n$ and $k=3$ for Intra-frame SICFSA. However, Figure 3 shows that the delay using Intra-frame SIC-FSA can be minimized when the number of replicas is a random number that follows the PDF in Equation (6) with $k=4$ and $m=1.5 n$. Under these conditions, Intra-frame SIC-FSA reduces the delay in a $55 \%$ with respect to FSA.

On the contrary, as it can be observed in Figure 3, the use of a random number of replicas in Inter-frame SIC-FSA does not reduce the delay with respect to fixed $k$. The minimum average delay using in Inter-frame SIC-FSA is reached with a fixed number of replicas $k=3$, with $m=1.5 n$ and $s=3$. Under these conditions, Inter-frame SIC-FSA reduces the delay in a $63 \%$ respect to FSA.

As it could be expected from the analytical delay and energy models, and as it can be observed in Figure 4, the coordinator energy consumption shows a similar trend to the average delay. Again, Intra-frame SIC-FSA minimizes the energy consumed by the coordinator when the number of replicas is random and follows the PDF with $k=4$ and $m=1.5 n$. Under these conditions, Intra-frame SIC-FSA reduces the energy consumed by the coordinator in a 55\% with respect to FSA. Inter-frame SIC-FSA provides up to a $63 \%$ of energy saving when the number of replicas is fixed in $k=3, m=1.5 n$ and $s=3$.

The energy consumed per end-device is represented in Fig-

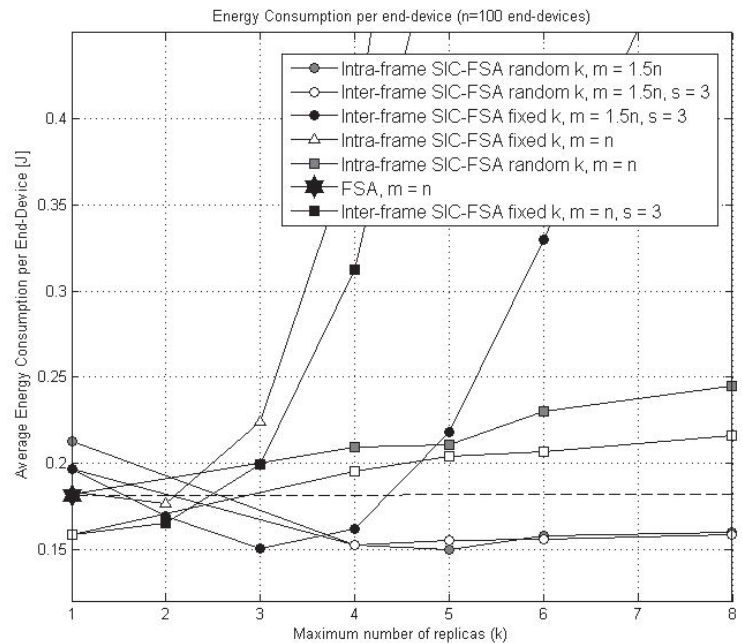

Figure 5. Average energy consumed per end-device using FSA, Intra-frame SIC-FSA and Inter-frame SIC-FSA over the maximum number of replicas.

ure 5. As it can be observed, Intra-frame SIC-FSA reaches its minimum value with a random number of replicas with $k=4$ and $m=1.5 n$. Under these conditions, the energy consumed per end-device is reduced in a $18 \%$ with respect to FSA. And finally, Inter-frame SIC-FSA improves the performance of FSA by decreasing the energy consumed per end-device in a $22 \%$ when the number of replicas is fixed with $k=3$ and $m=1.5 n$.

\section{B. Delay and Energy consumption in Dense M2M Networks}

In this section, we analyze the performance of Intra-frame SIC-FSA and Inter-frame SIC-FSA as a function of the number $n$ of end-devices. In all cases, we have selected the

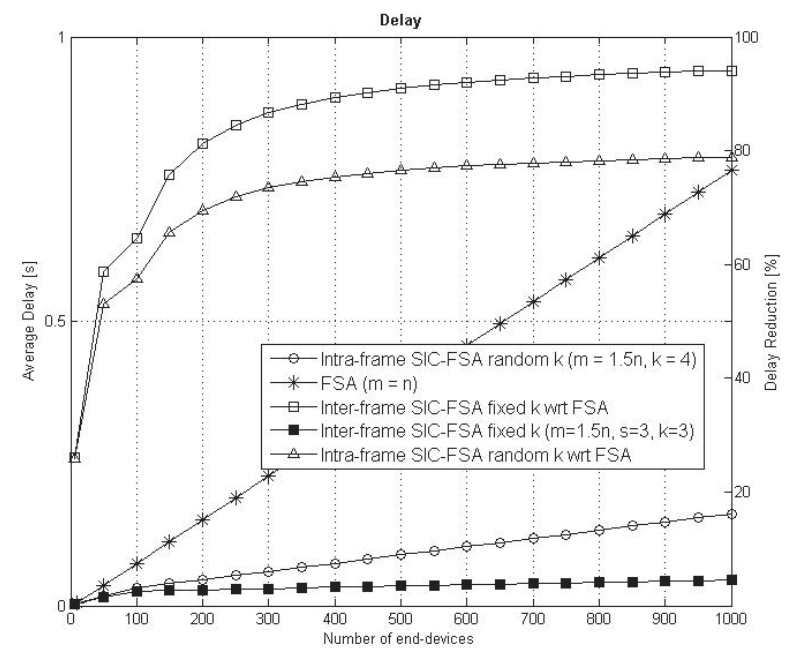

Figure 6. Average delay using FSA, Intra-frame SIC-FSA and Inter-frame SIC-FSA over the number of end-devices. 


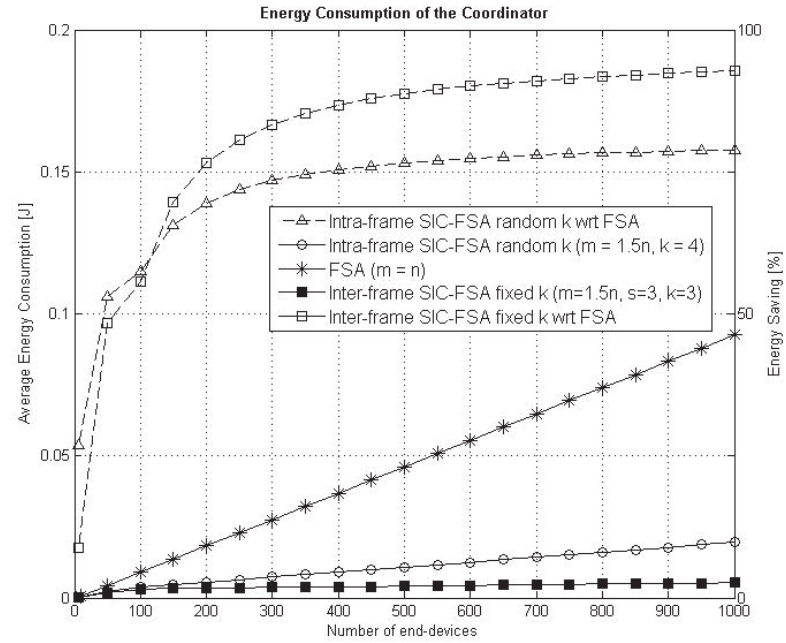

Figure 7. Average energy consumed by the coordinator using FSA, Intraframe SIC-FSA, and Inter-frame SIC-FSA over the number of end-devices.

optimum values of $k$ and $m$. According to the discussion of the previous section, this corresponds to: $(i)$ Intra-frame SIC-FSA with random $k=4$ and $m=1.5 n$; (ii) Inter-frame SIC-FSA with fixed $k=3, s=3$ and $m=1.5 n$; and (iii) FSA with $m=n[10]$.

Figures 6 and 7 show the average delay and the energy consumed by the coordinator. As it can be observed, the delay and the energy consumed by the coordinator increase almost linearly with the number of end-devices. As it could expected, Intra-frame and Inter-frame SIC-FSA outperform FSA in all cases. When $n<50$, both protocols produce similar results to FSA. However, when $n>500$, Intra-frame SIC-FSA reduces the average delay and energy consumption of the coordinator in a $79 \%$ with respect to FSA, while Inter-frame SIC-FSA provides a $94 \%$ of delay reduction and energy saving.

Figure 8 shows the energy consumed per end-device as a function of the number of end-devices involved in the data collection process. Although both Intra-frame and Inter-frame SIC-FSA reduce the energy consumption with respect to FSA when using their optimum configuration, Inter-frame SIC-FSA outperforms Intra-frame SIC-FSA. When $n>500$, Intra-frame SIC-FSA reduces the energy consumed per end-device in a $18 \%$ with respect to FSA, while Inter-frame SIC-FSA reduces in a $22 \%$ the energy consumed per end-device.

\section{Vi. Conclusion}

In this paper, we have evaluated the performance of the Intra-frame SIC-FSA and Inter-frame SIC-FSA protocols in data collection scenarios. We have evaluated the average delay and the average energy consumed by the end-devices and the coordinator. We have determined the optimum number of replicas and the optimum number of slots per frame to minimize delay and energy consumption. Considering the optimum configuration, results show that Intra-frame SIC-FSA reduces

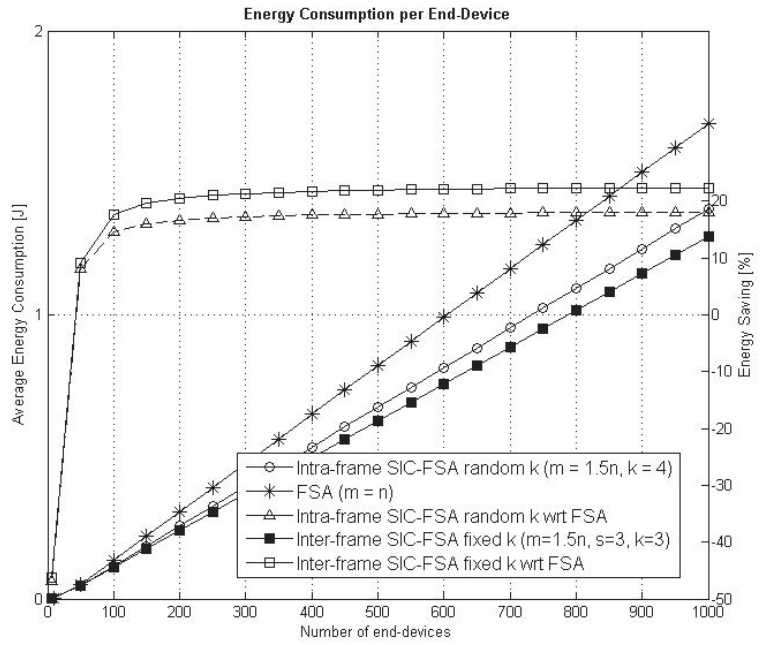

Figure 8. Average energy consumed per end-device using FSA, Intra-frame SIC-FSA, and Inter-frame SIC-FSA over the number of end-devices.

in more than a $75 \%$ the delay and the energy consumed by the coordinator with respect to FSA, and in a $18 \%$ the energy consumed per end-device. Inter-frame SIC-FSA reduces the delay and energy consumed by the coordinator in a $94 \%$ with respect to FSA, while the energy consumption per end-device is reduced in a $22 \%$. Future work aims at evaluating the performance analytically and at including the capture effect in the analysis.

\section{REFERENCES}

[1] E. Casini, R. De Gaudenzi, and O. Herrero, "Contention resolution diversity slotted aloha (CRDSA): An enhanced random access scheme for satellite access packet networks," IEEE Transactions on Wireless Communications, vol. 6, pp. 1408-1419, April 2007.

[2] G. Liva, "Graph-based analysis and optimization of contention resolution diversity slotted ALOHA," IEEE Transactions on Communications, vol. 59, pp. 477-487, February 2011.

[3] C. Stefanovic, K. Trilingsgaard, N. Pratas, and P. Popovski, "Joint estimation and contention-resolution protocol for wireless random access," in IEEE International Conference on Communications (ICC), pp. 33823387, June 2013.

[4] L. Toni and P. Frossard, "Prioritized random MAC optimization via graph-based analysis," CoRR, vol. abs/1501.00587, 2015.

[5] F. Vazquez-Gallego, M. Rietti, J. Bas, J. Alonso-Zarate, and L. Alonso, "Performance evaluation of frame slotted-aloha with succesive interference cancellation in machine-to-machine networks," in European Wireless 2014, pp. 1-6, May 2014.

[6] F. Ricciato and P. Castiglione, "Pseudo-random ALOHA for enhanced collision-recovery in RFID," IEEE Communications Letters, vol. 17, pp. 608-611, March 2013.

[7] P. Castiglione, F. Ricciato, and P. Popovski, "Pseudo-random Aloha for inter-frame soft combining in RFID systems," in International Conference on Digital Signal Processing, pp. 1-6, July 2013.

[8] IEEE Std., IEEE 802.11 Standard: Wireless LAN Medium Access Control (MAC) and Physical Layer (PHY) Specifications for Local and Metropolitan Area Networks, 2007.

[9] "RN131 datasheet." Available:http://www.rovingnetworks.com/products/ RN_131C_RN131C_RM.

[10] F. Vazquez-Gallego, L. Alonso, and J. Alonso-Zarate, "Energy and Delay Analysis of Contention Resolution Mechanisms for Machine-to-Machine Networks based on Low-Power WiFi," in IEEE International Conference on Communications (ICC), June 2013. 